\section{Antidepressant discontinuation and hypomania}

Dear Editor - Relapse of a known mood disorder following discontinuation of psychotropic medication is common. Discontinuation of maintenance antidepressant treatment in unipolar patients can lead to a relapse of depressive symptoms, ${ }^{1}$ and a manic relapse may result from discontinuation of mood-stabilising agent in bipolar patients..$^{2,3}$ It is however less common that sudden or tapered discontinuation of antidepressant medication may precipitate mania or hypomania. We present two patients with known diagnoses of bipolar affective disorder, who presented with depressive episodes, and subsequently became hypomanic following discontinuation of their antidepressant medication.

In both cases the antidepressant medication was being discontinued because of objective and subjective evidence of lack of efficacy. The management plan was to commence these patients on monoamine oxidase inhibitors but the hypomania occurred early on in the washout periods.

\section{Case reports}

PC, a 45-year-old gentleman with a known diagnosis of bipolar affective disorder - type 11, confirmed using a Structural Clinical Interview for DSM-IV ${ }^{4}$ was admitted with a protracted depressive episode. He was first diagnosed with BPAD after a hypomanic episode 15 years previously. $\mathrm{He}$ had four subsequent admissions with depressive episodes and outpatient visits showed that he had residual depressive symptomatology. Apart from the initial admission for hypomania, he had no subsequent admissions for manic/ hypomanic symptoms. He was admitted on this occasion on mirtazapine $15 \mathrm{mg}$ nocte and venlafaxine $37.5 \mathrm{mg}$ bd. He had been on this combination for two months. He had been unable to tolerate higher doses of either of these antidepressants. Antidepressants (fluoxetine and citalopram) and mood stabilisers (lithium, valproate and lamotrigine) had been tried previously with minimal effectiveness and significant associated side effects.

On admission the mirtazapine was discontinued. The venlafaxine was tapered down slowly over a two-week period. Two days after discontinuation of the venlafaxine his mood shifted. He became disinhibited, impulsive, restless, hyperactive, talkative and irritable, with symptoms worsening in the evening time. His sleep became even more disrupted with prominent initial insomnia. His speech was pressured, thoughts were racing, and his mood elated. This was initially observed in the hope that it may subside spontaneously.

Twenty days after the emergence of these hypomanic symptoms, he was commenced on carbamazepine. Over the subsequent month this was titrated upwards to a total of $100 \mathrm{mg}$ tid and his mood gradually settled.

$\mathrm{SC}$, a 50-year-old gentleman, with a diagnosis of bipolar affective disorder type 11, was admitted with severe depression with prominent biological features. He had been first diagnosed with depression eight years previously by his GP and was successfully treated with antidepressants at that time. Four years ago he experienced an episode of hypomania, was admitted to a psychiatric hospital, and his diagnosis was changed. In the year prior to this current admission his outpatient notes revealed that he had prominent depressive symptoms that were not responding to antidepressants and mood stabilisers. He admitted on citalopram $20 \mathrm{mg}$ od and lofepramine $70 \mathrm{mg}$ od. He had been on full doses of these agents individually previously.

Other psychotropic medications included quetiapine $200 \mathrm{mg}$ nocte and lamotrigine $100 \mathrm{mg}$ bd. He had undertaken trials of other mood stabilisers, such as lithium and valproate, in the past with some initial improvement, which, unfortunately, was not sustained. Both the citalopram and the lofepramine were tapered down over a seven-day period.

Within three days of complete antidepressant discontinuation his mood switched to one of elation, with prominent grandiosity, irritability, racing thoughts, irresponsible spending, excessive and unrealistic plan-making activity and a generalised over activity. This was treated by reduced stimulation, an increase in his quetiapine to $400 \mathrm{mg} \mathrm{bd}$, and addition of chlorpromazine $100 \mathrm{mg}$ bd (a combination which was previously effective). Over the next two weeks his elation subsided.

Both of our case studies illustrate the development of hypomania following discontinuation of antidepressants in patients with known diagnoses of bipolar affective disorder type 11. The first report of antidepressant - withdrawal mania and hypomania in the literature was probably that of Mirin et $a l .{ }^{5}$ They observed the induction of mania in seven remitted depressed patients two to seven days after the discontinuation of tricyclic antidepressants. In their report patients treated with neuroleptics improved in five to nine days and patients receiving no treatment experienced symptoms for 14-28 days.

The most dramatic aspect of both of our cases was that the mood switch from depression to hypomania occurred relatively quickly, over the course of four days. It could be suggested that the syndrome we observed was the result of a naturally occurring switch from depression to hypomania, however the short time span from stopping the antidepressant and the emergence of the hypomanic symptoms would strongly suggested that they were directly related. This is particularly relevant in our cases, as they had spent a protracted amount of time in depressive states, especially in the 12 months previously. This short time span between antidepressant discontinuation and hypomanic symptoms has been shown before..$^{5-7}$

One could argue that these are not true hypomanic episodes triggered by the antidepressant withdrawal and are instead withdrawal phenomena. Our patents had been withdrawn from antidepressants previously and there was no recollection of withdrawal symptoms or documentation of same in their notes. The gradual tapering of the antidepressants undertaken in both cases was to lessen any potential withdrawal effects and it is important to point out that the hypomanic symptoms only emerged after complete discontinuation of the antidepressants rather than during the tapering period. The type of symptoms experienced (pressure of speech, grandiose ideas, etc.) would also point away from withdrawal symptoms. 
In summary we have discussed two patients who developed acute hypomanic symptoms after tapering of their antidepressant treatments. Our cases developed symptoms while inpatients. One would wonder how often this would occur on an outpatient basis and would these patients be aware of the mood switch and present for further treatment. These cases highlight the need for vigilance and close monitoring of the patient with BPAD on discontinuation of antidepressants, particularly in the first four days for the possibility of antidepressant withdrawal induced hypomania.

*Marie Naughton

Registrar in Psychiatry, St James's Hospital, Dublin 8

Darren Roddy,

Registrar in Psychiatry, St Edmundsbury Hospital,

Lucan, Co Dublin,

John Cooney,

Consultant Psychiatrist,

St James's Hospital, Dublin 8, Ireland.

${ }^{\star}$ Correspondence

\section{References}

1. Kupfer $D$ et al. Five-year outcome for maintenance therapies in recurrent depression. Arch Gen Psychiatry 1992; 49: 769-73.

2. Post RM et al. Prophylactic efficacy of carbamazepine in manic-depressive illness. Am J Psychiatry 1983; 140: 1602.04.

3. Suppes $T$ et al. Risk of recurrence following discontinuation of lithium treatment in bipolar disorder. Arch Gen Psychiatry 1991; 48:1082-88.

4. First MB, Spitzer RL, Gibbon M et al. Structured Clinical Interview for DSM-IV Axis 1 Disorders-patient edition (SCID-I/P, Version 2.0). New York, NY: Biometric Research, New York State Psychiatric Institute: 1995.

5. Mirin SM, Schatzberg AF, Creasey DE. Hypomania and mania after withdrawal of tricyclic antidepressant. Am J Psychiatry 1981; 138: 87-89.

6. Steven M, Mirrin MD, Schatzberg MD, David E, Creasey MD. Hypomania and mania after withdrawal of tricyclic antidepressants. Am J Psychiatry 1981; 138: 87-89.

7. Dilsaver SC, Greden JF. Antidepressant withdrawal Phenomena. Biol Psychiatry 1984: $19: 237-56$

8. Chittaranjan A. Antidepressant - withdrawal mania: a critical review and synthesis of the literature. 1 Clin Psychiatry 2004; 65: 987-93.

\section{Discontinuation syndrome en masse}

Dear Editor - Every psychiatrist will have at least one. The patient who had a psychosis that blazed for years, apparently untreatable, until thioridazine quelled the flames. Then things stabilised for some years. Until thioridazine (or the tranylcypromine/trifluoperazine combination Parstelin, or whichever now discontinued drug) was withdrawn, or withdrawal was threatened. "They've never been right since," the CPN or hostel CNM will say. Often the only answer seems to recommence the discontinued drug, if possible. This then continues until production is ceased. Thioridazine has been dancing on the volcano of complete discontinuation for what seems like forever. Carers, professionals and patients themselves look forward with apprehension to the day of its complete disappearance.

Drugs are discontinued for a range of reasons. Some are withdrawn because of safety concerns, such as both of the examples above. Others have their production ceased for economic reasons. Some preparations are only made by the smaller companies of the pharmaceutical industry. These of course are more vulnerable to the vicissitudes of commerce.

The association of some older medications with sudden death and prolonged QTc interval is well known. ${ }^{1}$ Newer medications, of course, increasingly are known to have associations with deleterious physical outcomes. Olanzapine, for instance, is diabetogenic and associated with obesity and resultant increase in lipid profile.

It is important that the safety of medications is rigorously policed by regulatory bodies. Psychiatric patients deserve, as much as any other patient group, to have their physical health secure from the medications supposed to help them.

In the case of psychotropic medication, however, there is an issue of a stability from a psychiatric point of view. It can often be difficult to find the medication or the right combination of medication to stabilise a particular individual. In researching this issue, there is an absolute paucity of work on the psychological effects on patients and their carers of drug discontinuation, as well as the direct effect on mental state on the withdrawal of a previously effective medication. The psychological effect of discontinuation does not seem to be taken into account by regulatory bodies. The makers of thioridazine are unaware of any research on the psychological impact of the discontinuation of the drug. ${ }^{2}$

There is an assumption that withdrawal of one agent can simply be compensated for by replacement with another, newer one. Clinical experience - such as the patient "who was only ever right on Melleril" discussed above - tells us otherwise. Each patient is an individual, whose response to any particular agent or combination of agents in the pharmacopoeia is individual and - until, perhaps, the genetic basis of neurotransmitter regulation is a more exact science unpredictable.

On a population basis new atypical antipsychotic $A$ may be more efficacious and less 'dirty' (although give it time) than venerable typical antipsychotic $B$, but the primary duty of any doctor is to the individual patient across the desk or in the bay bed.

Patient advocacy groups both here and in the UK are greatly concerned with the minimisation of side effects and, when necessary, the withdrawal of potentially hazardous medications. ${ }^{3}$ This is of course laudable and extremely necessary. However one wonders how much suffering has been caused by the abrupt and enforced discontinuation of these drugs.

With many drugs with an adverse side effect profile, availability on a named patient basis continues. Thalidomide, which is in the public mind the most poignant and resonant example of a drug with disastrous side effects, has made something of a comeback, with a recognition that it is an effective treatment for multiple myeloma, discoid lupus erythematosus, leprosy and certain dermatological conditions. ${ }^{4}$

Could not psychotropic medications that have a similar track record of effectiveness be made available, on a named patient basis and under stringent safeguards, rather than being abruptly discontinued? Patients too could and should be involved in the decision. There is something of a misapprehension that psychiatric patients are simply unable to 